\title{
Um visitante inesperado: pelicanos-pardos (Pelecanus occidentalis) e suas interações com pescadores artesanais no Parque Nacional de Anavilhanas
}

An unexpected visitor: brown pelicans (Pelecanus occidentalis) and their interactions with artisanal

\author{
fishermen in the Anavilhanas National Park
}

Un visitante inesperado: pelícanos pardos (Pelecanus occidentalis) y sus interacciones con

pescadores artesanales en el Parque Nacional de Anavilhanas

Recebido: 10/02/2022 | Revisado: 16/02/2022 | Aceito: 23/02/2022 | Publicado: 04/03/2022

\author{
Marcelo Derzi Vidal \\ ORCID: https://orcid.org/0000-0002-9434-7333 \\ Instituto Chico Mendes de Conservação da Biodiversidade, Brasil \\ E-mail: marcelo.vidal@icmbio.gov.br \\ Fábio Pereira da Conceição \\ ORCID: https://orcid.org/0000-0002-3029-2388 \\ Instituto Australis de Pesquisa e Monitoramento Ambiental, Brasil \\ E-mail: Fabio_pconceicao@hotmail.com
}

\begin{abstract}
Resumo
O pelicano-pardo (Pelecanus occidentalis) é uma ave marinha com ampla distribuição geográfica pela região costeira do continente americano. A espécie vive a maior parte da sua vida no mar, retornando ao continente apenas para nidificar. O presente trabalho relata a ocorrência de pelicanos-pardos no Parque Nacional de Anavilhanas, Amazônia brasileira, e descreve como pescadores artesanais que residem no entorno desta Unidade de Conservação vêm interagindo com este visitante inesperado. Por meio de avistamentos em campo e entrevistas com moradores locais, foram registrados até seis pelicanos-pardos que, segundo moradores locais, apareceram pela primeira vez no segundo semestre de 2019. As interações entre os pescadores e as aves envolvem especialmente a oferta de peixes, mas também foram registrados conflitos, como quando os pelicanos são atraídos pelo cheiro do peixe e vão em direção às embarcações de pesca, fazendo com que os pescadores tenham que afugentar as aves, e o relato de abate de uma das aves para consumo. Este é o registro mais interior da espécie em ambiente continental amazônico e o primeiro a descrever um agrupamento de pelicanos-pardos em interação com moradores da região. Os resultados apresentados demonstram que pouco sabemos sobre os fatores que influenciam a migração e dispersão dos pelicanos-pardos, contribuem para um melhor entendimento de atitudes culturais relacionadas às interações humano-fauna, e subsidiam o manejo conservacionista destas aves pouco comuns em áreas interiores amazônicas.
\end{abstract}

Palavras-chave: Amazônia; Conflitos; Fauna silvestre; Oferta alimentar.

\begin{abstract}
The brown pelican (Pelecanus occidentalis) is a seabird with wide geographic distribution along the coast of the American continent. The species lives most of its life at sea, returning to the mainland only to nest. This paper reports the occurrence of brown pelicans in the Anavilhanas National Park, Brazilian Amazon, and describes how artisanal fishermen living in the surroundings of this Conservation Unit have been interacting with this unexpected visitor. Through field sightings and interviews with local residents, up to six brown pelicans were recorded which, according to local residents, appeared for the first time in the second half of 2019. The interactions between fishermen and birds involve especially the offer of fish, but conflicts were also recorded, such as when pelicans are attracted by the smell of fish and head towards fishing vessels, causing fishermen to chase the birds away, and the report of the slaughter of one of the birds for consumption. This is the most interior record of the species in a continental Amazonian environment and the first to describe a cluster of brown pelicans interacting with inhabitants of the region. The results presented demonstrate that we know little about the factors that influence the migration and dispersion of brown pelicans, contribute to a better understanding of cultural attitudes related to human-fauna interactions, and support the conservation management of these rare birds in interior Amazonian areas.
\end{abstract}

Keywords: Amazon; Conflicts; Wildlife; Food offer.

\section{Resumen}

El pelicano pardo (Pelecanus occidentalis) es un ave marina con amplia distribución geográfica a lo largo de la costa del continente americano. La espécie vive la mayor parte de su vida en el mar y regresa al continente solo para anidar. Este artículo describe la ocurrencia de pelícanos pardos en el Parque Nacional Anavilhanas, Amazonía brasileña, y 
describe cómo los pescadores artesanales que viven en los alrededores de esta Unidad de Conservación han estado interactuando con este visitante inesperado. Através de avistamientos de campo y entrevistas con moradores locales, se registraron hasta seis pelícanos pardos que aparecieron por primera vez en el segundo semestre de 2019. Las interacciones entre pescadores y aves involucran especialmente la oferta de pescado, pero conflitos también se registraron, como cuando los pelícanos son atraídos por el olor del pescado y se dirigen hacia los barcos de pesca, lo que provoca que los pescadores ahuyenten a las aves, y el informe de la matanza de una de las aves para su consumo. Este es el registro más interior de la especie en un ambiente amazónico continental y el primero en describir un grupo de pelícanos pardos que interactúan con los habitantes de la región. Los resultados presentados demuestran que sabemos poco sobre los factores que influyen en la migración y dispersión de los pelícanos pardos, contribuyen a una mejor comprensión de las actitudes culturales relacionadas con las interacciones humano-fauna y apoyan el manejo de la conservación de estas raras aves en las áreas del interior amazónico.

Palabras clave: Amazonía; Conflictos; Fauna silvestre; Suministro de alimentos.

\section{Introdução}

O pelicano-pardo (Pelecanus occidentalis) é uma ave marinha da ordem Pelecaniformes, com ampla distribuição geográfica pelo continente americano, especialmente em áreas costeiras (Almeida-Santos et al., 2015). Sua ocorrência vai desde os Estados Unidos até o norte da América do Sul, com visitas esporádicas até a Terra do Fogo, no extremo sul do continente (Enticott et al., 1997; Sick, 1997).

Considerado o menor dos pelicaniformes, $P$. occidentalis mede de 105 a $188 \mathrm{~cm}$, podendo atingir até $2 \mathrm{~m}$ de envergadura e pesar de 2,7 a $15 \mathrm{~kg}$ (Grzimek, 2002). Os adultos têm plumagem principalmente cinza e marrom, com um bico acastanhado, bolsa cinza na garganta e pele facial e pernas pretas (Shields, 2002; Peterson, 2008; Tangley, 2009). Durante a época de reprodução os indivíduos apresentam uma coloração que varia de castanho a cinza e branco (Vargas-Masís et al., 2014).

Os pelicanos-pardos são coloniais, com algumas agregações sendo mantidas por vários anos, nidificam principalmente no chão, às vezes em penhascos e, com menos frequência, em pequenas árvores ou arbustos. A época de reprodução varia de acordo com a latitude, reproduzindo na primavera no extremo norte de sua distribuição, em comparação com o ano todo nos trópicos (Shields, 2002).

Sendo uma ave extremamente adaptada ao ambiente marinho, o pelicano-pardo vive a maior parte da sua vida no mar, retornando ao continente apenas para nidificar. Sua dieta é composta principalmente de peixes, como sardinhas e anchovas, mas também há registros de consumo de camarão, carniça e até filhotes de garça (Del Hoyo et al., 1992).

A Lista Vermelha da União Internacional para Conservação da Natureza classifica o pelicano-pardo na categoria Menos Preocupante (Birdlife International, 2018), apesar de ao longo de sua distribuição a espécie sofrer diversas ameaças, como lesões causadas por emalhamento em linhas de pesca e anzóis (Franson et al., 2003) e turismo desordenado em colônias de reprodução, levando principalmente a perdas e abandono de ninhos (Elliott et al., 2018).

Movimentos e migrações de pelicanos-pardos dependem das condições ambientais locais, sendo comuns as migrações de indivíduos do Hemisfério Norte para o Hemisfério Sul (Del Hoyo et al., 1992). No Brasil, o pelicano-pardo é considerado um visitante eventual. Em áreas costeiras há registros na Baía de Guanabara, estado do Rio de Janeiro (Mitchell, 1957), Baía de Todos os Santos, estado da Bahia (Teixeira et al., 1993), e na praia de Ipióca, estado de Alagoas (Patrial et al., 2011).

$\mathrm{O}$ estudo das interações e associações entre pessoas e animais pode demonstrar tanto valores e significados culturais que diferenciam comunidades humanas em relação às espécies da fauna, quanto o conhecimento que estas comunidades apresentam sobre áreas de ocorrência e padrões comportamentais de algumas espécies animais (Alves et al., 2018; Filgueira et al., 2021). Tal conhecimento etnozoológico é importante quando considerados no desenvolvimento de planos de conservação em longo prazo que não somente protejam a fauna silvestre, mas também sustentem as crenças e práticas de comunidades locais (Alves et al., 2015). 
A América do Sul é considerada o continente das aves, pois apresenta em seu território cerca de um terço de todas as espécies de aves do planeta (Sick, 1997). Neste continente, áreas de elevada diversidade biológica e cultural frequentemente coincidem (Costa-Neto et al., 2009). No entanto, para a Amazônia, a grande variação espacial dos esforços de coleta e a pequena proporção amostrada até o momento, mesmo para os táxons mais conhecidos, sugere um desconcertante desconhecimento da região (Peres, 2005). Consequentemente, temos grandes dificuldades em planejar a adequada conservação e uso sustentável da sociobiodiversidade (Vidal et al., 2019).

O presente trabalho relata a ocorrência de pelicanos-pardos no Parque Nacional de Anavilhanas, Amazônia brasileira, e descreve como pescadores artesanais que residem no entorno desta Unidade de Conservação vêm interagindo com este visitante inesperado. Este é o registro mais interior da espécie em ambiente continental amazônico e o primeiro a descrever um agrupamento de pelicanos-pardos em interação com moradores da região. Os resultados apresentados podem contribuir para um melhor entendimento de atitudes culturais relacionadas às interações humano-fauna, bem como subsidiar o manejo conservacionista destas aves pouco comuns em áreas interiores amazônicas.

\section{Metodologia}

\subsection{Caracterização da área de estudo}

O Parque Nacional de Anavilhanas é uma Unidade de Conservação de proteção integral gerenciada pelo Instituto Chico Mendes de Conservação da Biodiversidade (ICMBio). Situado em frente à cidade de Novo Airão (Figura 1), no estado do Amazonas, o Parque tem área aproximada de 350 mil hectares e abrange cerca de 400 ilhas, o que o torna o segundo maior arquipélago fluvial do mundo (Brasil, 2011).

Figura 1. Mapa do Baixo Rio Negro, identificando a cidade de Novo Airão e a área do Parque Nacional de Anavilhanas.

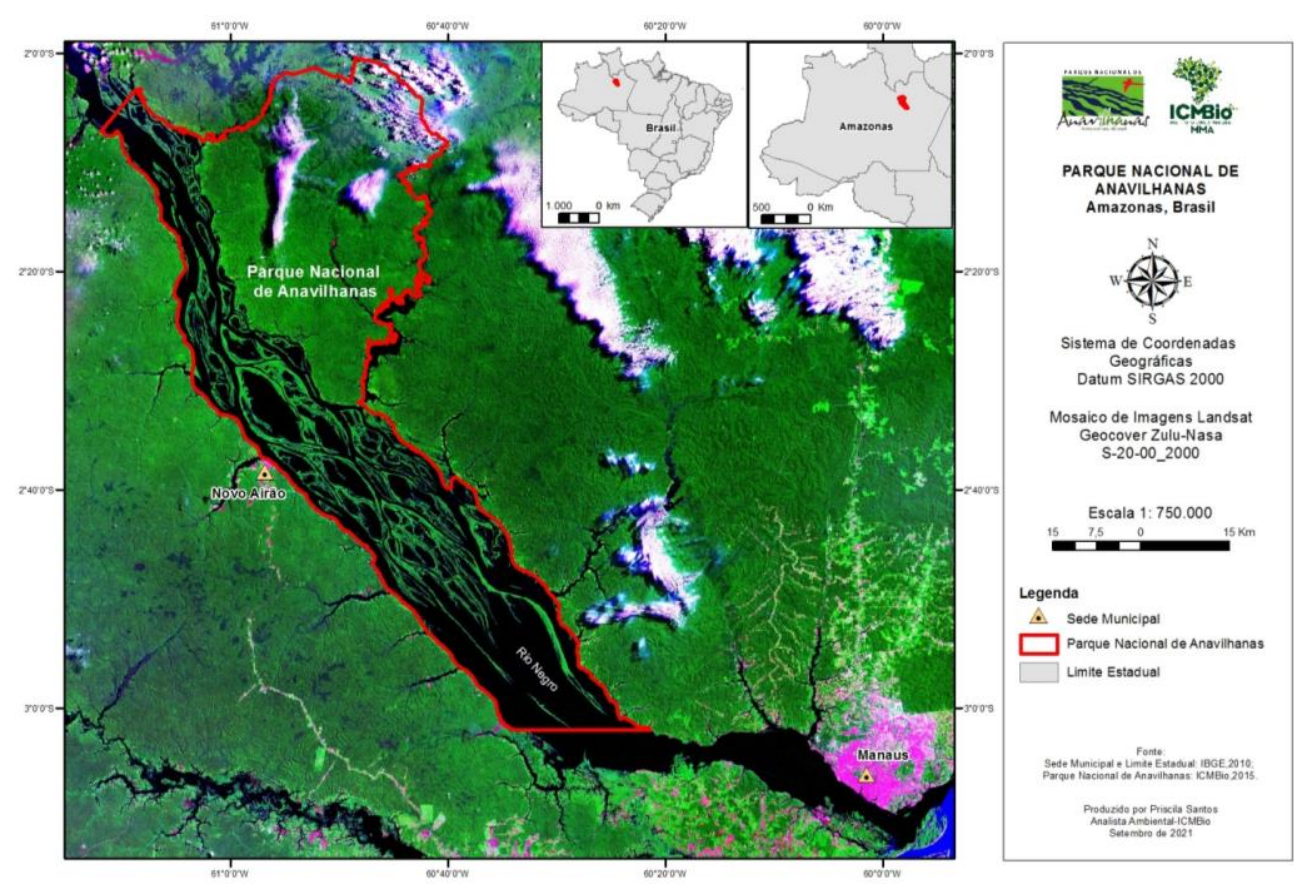

Fonte: Santos, P.M.C (2021).

A cidade de Novo Airão localiza-se na margem direita do rio Negro, distante $183 \mathrm{~km}$ por via terrestre de Manaus, capital do Amazonas. Em função de sua proximidade com Manaus e a facilidade de acesso por via terrestre pavimentada, Novo Airão é um dos principais destinos turísticos para os que visitam a Amazônia, e para os habitantes de Manaus e cidades 
próximas, sobretudo por seus atrativos naturais (Vidal et al., 2017). As principais atividades de visitação no Parque incluem a interação com botos, passeios em trilhas aquáticas, atividades náuticas, visita e pernoite em praias, caminhadas em trilhas terrestres, voo panorâmico, escalada em árvores, e observação da fauna e da flora locais (Silva et al., 2020).

\subsection{Coleta de dados}

Em março de 2020, foram realizadas entrevistas com quatro moradores locais de Novo Airão (dois pescadores, um condutor de turismo e um gestor do Parque Nacional de Anavilhanas) que trabalhavam e/ou moravam próximos aos pontos onde os pelicanos-pardos foram registrados. Salienta-se que, em pesquisas qualitativas sobre percepção, novos relatos podem não apresentar novas informações relacionadas aos objetivos do estudo e com isso os dados podem se tornar repetitivos (Mason, 2010). Por isso, frequências de tamanho amostral grande raramente são importantes em pesquisas envolvendo etnobiologia e percepção (Crouch et al., 2006), o que justifica o tamanho amostral neste estudo.

As entrevistas foram guiadas por um roteiro semiestruturado, contendo perguntas abertas e fechadas que abordaram informações relacionadas aos animais (número de indivíduos, quando chegaram na região, de que se alimentavam, onde dormiam, que horas iniciavam suas atividades diárias, existência de interações positivas ou conflitos com moradores locais). As entrevistas foram realizadas individualmente com os entrevistados para evitar interferência de outros atores que pudessem estar nas proximidades (Schensul et al., 1999) e, para obter fidelidade nos relatos e estabelecer comparações entre as informações obtidas (Silva et al., 2015), foi utilizada a técnica de informações repetidas em situação sincrônica, em que o mesmo roteiro foi aplicado a todos os entrevistados (Goldenberg, 1999; Opdenakker, 2006). O suporte metodológico para o modelo de pesquisa aqui apresentado, de natureza quali-quantitativa, considerou ainda a bibliografia especializada sobre métodos de pesquisa científica e social (Gil, 2008; Pereira et al., 2018).

A coleta de dados em campo foi complementada por meio de duas visitas ao local em que os pelicanos-pardos costumavam ser vistos (Porto do Padre, entorno imediato do Parque Nacional de Anavilhanas) em interação com moradores locais. Os dados primários, coletados em campo, foram ainda complementados e discutidos com dados secundários extraídos de publicações científicas (aqui considerados artigos científicos, livros e capítulos) encontradas na rede mundial de internet.

\section{Resultados}

Em novembro de 2019, seis pelicanos-pardos foram avistados sobrevoando o canal principal do rio Negro, no interior do Parque Nacional de Anavilhanas. Posteriormente, em fevereiro e março de 2020, foram registrados quatro e três indivíduos, respectivamente, no Porto do Padre, situado no igarapé Freguesia, entorno imediato da área protegida (Figura 2).

Devido os primeiros animais terem sido avistados em deslocamento aéreo, não foi possível a realização do registro fotográfico. Por outro lado, após observação dos indivíduos avistados no Porto do Padre e a análise dos seus respectivos registros fotográficos, concluiu-se que todos os indivíduos eram juvenis (Figura 3), já que apresentavam cabeça, pescoço e partes superiores marrons com pontas castanho-claro, ventre branco, graduando-se em marrom acinzentado nas laterais, asas marrons acinzentado com pontas brancas, padrão característico de indivíduos nessa faixa etária (Shields, 2002). 
Figura 2. Mapa de localização dos registros de pelicanos-pardos no Parque Nacional de Anavilhanas e seu entorno imediato.
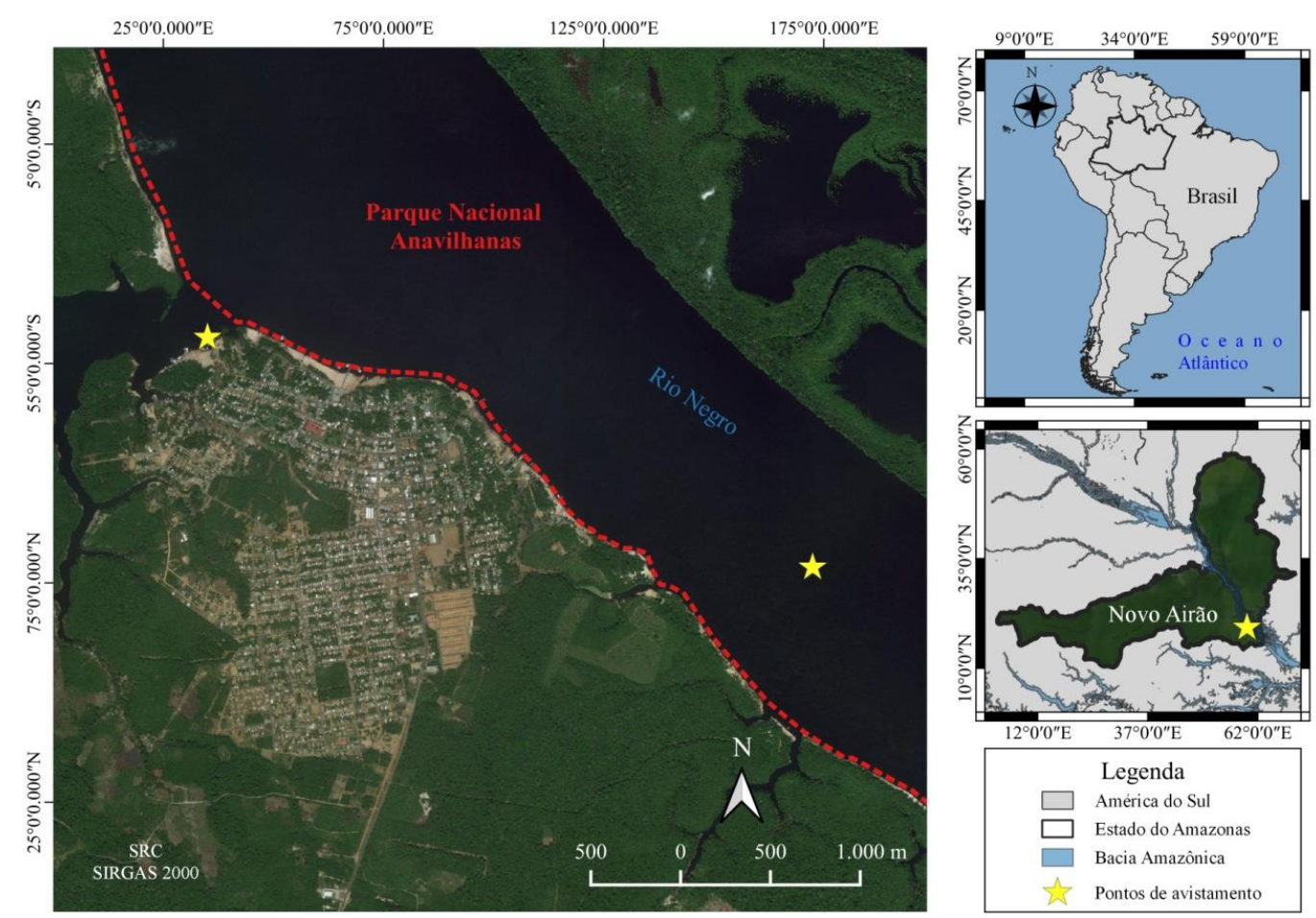

Fonte: Toffolo, C. (2022).

Figura 3. Pelicanos-pardos registrados em fevereiro (A) e março (B) de 2020 no Porto do Padre, entorno imediato do Parque Nacional de Anavilhanas.

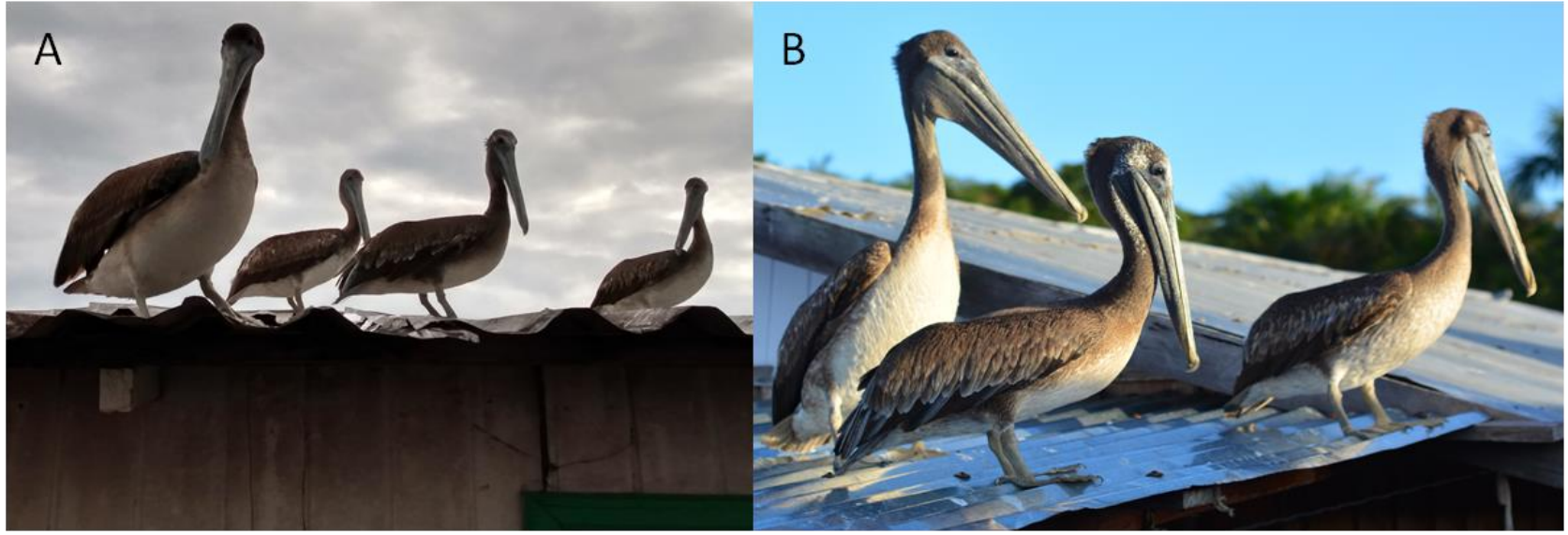

Fonte: Autores (2022).

Não foi possível identificar o sexo dos indivíduos, já que em pelicanos-pardos o dimorfismo sexual se dá apenas pelo tamanho dos indivíduos adultos, onde os machos tendem a serem maiores do que as fêmeas (Shields, 2002).

De acordo com um dos entrevistados, morador da região do Porto do Padre, os pelicanos-pardos apareceram pela primeira vez em janeiro de 2020. No entanto, outro morador da região declarou que os animais apareceram entre setembro e outubro de 2019. De acordo com este entrevistado, inicialmente eram avistados seis animais, mas, depois de um tempo, somente quatro indivíduos passaram a serem vistos na área.

Os entrevistados afirmaram que diariamente, por volta das 18:00 horas, os pelicanos-pardos deslocam-se em direção à floresta no interior do Parque Nacional para dormir, retornando ao Porto do Padre no dia seguinte, aproximadamente às 06:00 horas, onde permanecem o dia inteiro. 
Um dos entrevistados afirmou alimentar os pelicanos-pardos (Figura 4) duas vezes ao dia. "Dou peixes para os pelicanos por volta das 08:00 horas e outra vez no final da tarde, aproximadamente às cinco horas". Segundo o entrevistado, os peixes ofertados às aves são espécies pescadas ou compradas na própria região, especialmente piranha Serrasalmus spp., sardinha Triportheus spp. e aracu Leporinus spp. Segundo o gestor do Parque Nacional de Anavilhanas, as interações envolvendo os pescadores e os pelicanos vêm sendo monitoradas e orientações são repassadas aos pescadores, inclusive com a orientação de não se ofertar alimentos e evitar o contato direto com as aves.

Figura 4. Pescador ofertando peixe aos pelicanos-pardos (A); um dos animais alimentando-se do peixe ofertado (B) no Porto do Padre, entorno imediato do Parque Nacional de Anavilhanas.

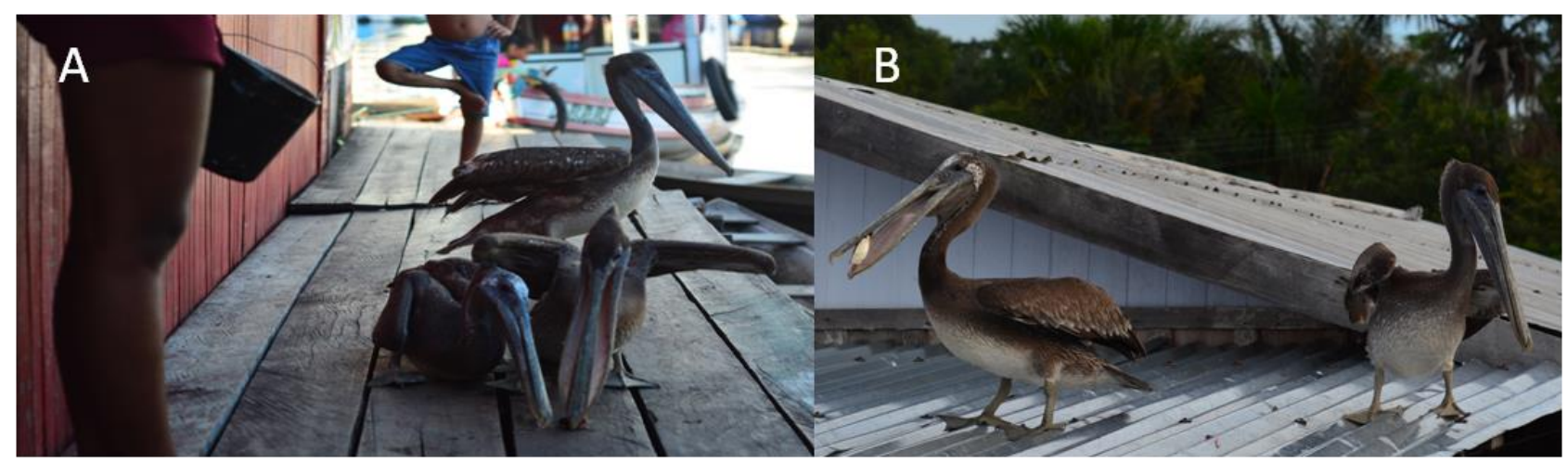

Fonte: Autores (2022).

Todos os entrevistados acreditam que as aves sobrevivem da alimentação ofertada pelas pessoas, dos restos de peixes descartados na água por pescadores, e dos peixes que podem ativamente capturar; apesar de terem declarado não saber exatamente quais peixes as aves capturam e que nunca as viram capturando ativamente suas presas.

A existência de conflitos envolvendo os pelicanos-pardos e os pescadores locais foi também relatada. Segundo um dos entrevistados, durante as atividades de pesca desenvolvidas pelos pescadores, ou mesmo quando estes chegam de suas atividades pesqueiras, as aves são atraídas pelo cheiro do peixe e vão em direção às embarcações, fazendo com que os pescadores tenham que afugentar as aves. "A gente grita e bate pedaços de madeira ou o remo na água para espantar os pelicanos". Um dos entrevistados afirmou que um pescador local abateu um dos pelicanos, em retaliação à sua interferência na pesca, e se alimentou do animal. "Ele (o pescador) matou o pelicano e cozinhou para comer; mas o bicho era muito pitiú (tinha cheiro/sabor de peixe)".

\section{Discussão}

Sendo uma ave essencialmente marinha, registros de pelicanos-pardos são escassos em áreas interiores da Amazônia brasileira. O primeiro registro da espécie na região foi em 1914, no rio Tapajós, cidade de Itaituba, estado do Pará, quando um indivíduo foi coletado e depositado no Museu Paraense Emílio Goeldi, em Belém (Snethlage, 1914). A partir de então, outros quatro avistamentos de pelicanos-pardos na Amazônia brasileira foram descritos em revistas científicas ou livros, mas todos registrando somente um indivíduo em cada localidade (Tabela 1). 
Tabela 1. Registros de ocorrência de pelicanos-pardos (Pelecanus occidentalis) em áreas interiores da Amazônia brasileira.

\begin{tabular}{lccc}
\hline \multicolumn{1}{c}{ Rio } & Município/Estado & $\mathrm{N}^{0}$ de indivíduos & Referência \\
\hline Tapajós & Itaituba/PA & 1 & SNETHLAGE (1914) \\
Uraricoera & Não informado/RR & 1 & PINTO (1978) \\
Branco & Não informado & Não informado & SICK (1997) \\
Tapajós & Santarém/PA & 1 & ALMEIDA-SANTOS et al. (2015) \\
Trombetas & Oriximiná/PA & 1 & GOMES et al. (2016) \\
Negro & Novo Airão/AM & 6 & Estudo atual \\
\hline
\end{tabular}

Fonte: Autores (2022).

Pode-se classificar como instigante e inesperado o registro de pelicanos-pardos na região de Novo Airão. Gomes et al. (2016) sugerem que os pelicanos-pardos avistados no rio Uraricoera, Roraima, possam ter vindo da costa da Guiana cruzando pela foz do rio Essequibo, ou mesmo vindo pelos rios Amazonas e Branco, configurando assim uma maior distância percorrida. Ainda que consideremos uma linha perpendicular com o ponto mais próximo do litoral da América do Sul, situado na Guiana, os indivíduos registrados em Novo Airão terão percorrido aproximadamente 1.000 quilômetros fora de sua conhecida rota migratória na costa sul-americana, classificando assim o registro atual da espécie como o mais interior em ambiente continental amazônico e o primeiro a descrever um agrupamento de indivíduos em interação com moradores da região.

Sendo aves predadoras de topo tipicamente aquáticas, os pelicanos obtêm seu alimento nesses ambientes, influenciando a dinâmica populacional de suas presas e indiretamente relacionando-se com a atividade pesqueira (Mazzochi \& Carlos, 2020). A dieta do pelicano-pardo é composta principalmente de peixes, como sardinhas e enchovas, mas também podem se alimentar de outros animais, como camarões, moluscos e pequenas aves, como filhotes de garça (Del Hoyo et al., 1992; Shields, 2002). No entanto, o tipo de presa preferida dos pelicanos depende da localização onde os animais estão inseridos. Por exemplo, ao longo das costas do Atlântico e do Golfo dos EUA, pelicanos geralmente comem savelha (Brevoortia spp.) e tainha (Mugil spp.) espécies comuns na região (Palmer, 1962; Blus et al., 1979; Fogarty et al., 1981). Já na região do Caribe, preferem o arenque-anão (Jenkinsia lamprotaenia), anchova (Anchoa spp., Engraulis spp.) e sardinha (Sardinella spp., Harengula spp.) (Voous, 1983; Collazo, 1985). O fato dos pelicanos-pardos capturarem suas presas por meio de mergulhos pode justificar o fato dos pescadores de Novo Airão não saberem informar quais espécies as aves capturam, mas surpreende o fato de os pescadores nunca terem visto o comportamento de mergulho executado para capturar as presas.

No rio Negro, onde está inserido o Parque Nacional de Anavilhanas, há uma grande diversidade de peixes, a pesca ocorre dentro e fora da Unidade de Conservação, e normalmente os pescadores preferem espécies como o acará (Astronotus spp.), a dourada (Blachyplatystoma rosseauxii), o surubim (Pseudoplatystoma fasciatum), o tucunaré (Cichla spp.), o pacu (Myleus spp. e Mylossoma spp.), a matrinchã (Brycon spp.) e o jaraqui (Semaprochilodus spp.) (Goulding et al., 1988; Illenseer \& Pereira, 2011). Nesta região, os pelicanos-pardos vêm sendo ativamente alimentados por moradores locais com peixes que são facilmente capturados na região, mas geralmente menos valorizados no consumo humano (como a piranha e o aracu) e, por consequência, mais baratos comercialmente. Apesar da imensa riqueza de peixes amazônicos, estimada entre 1.500 e 3.000 espécies, somente cerca de 100 destas são exploradas comercialmente pela pesca, sendo que aproximadamente $90 \%$ estão concentrados somente em uma dezena de espécies, destacando-se o tambaqui (Colossoma macropomum), o curimatã (Prochilodus nigricans), o jaraqui, a matrinchã, o pacu e o tucunaré (Santos et al., 2006).

A interação dos pelicanos-pardos com as embarcações e artefatos de pesca na região do rio Negro é preocupante. Nesta região os pescadores utilizam uma variedade de petrechos de pesca, como arpão, caniço, espinhel, tarrafa e malhadeira (Rodrigues, 2014). Alguns destes artefatos, como os espinhéis e as redes de emalhar, estão entre os mais envolvidos na captura 
incidental de animais aquáticos que não são alvos das pescarias, o que levou nas últimas décadas a um frequente registro de capturas não intencionais de mamíferos aquáticos e aves (Bugoni et al., 2008; Ferreira et al., 2010; Mazzochi \& Carlos, 2020). $\mathrm{Na}$ verdade, a captura incidental pela pesca é considerada a maior ameaça enfrentada por muitas populações de aves marinhas (Birdlife International, 2014). Além disso, causa prejuízo tanto para as aves, já que geralmente ocasiona sua morte, quanto para o pescador, uma vez que o consumo das iscas pelas aves (no caso do espinhel) e o emalhamento das aves (no caso das redes) diminuem a capacidade produtiva dos artefatos (Neves et al., 2006). Sabe-se ainda que algumas aves interagem diretamente com embarcações pesqueiras, seguindo-as em busca de espécies não alvo da pesca que são descartadas (Prince \& Morgan, 1987; Vooren \& Fernandes, 1989). Esse é o caso do pelicano-peruano ( $P$. thagus), uma espécie que se associa comumente a barcos, principalmente por causa do descarte de pesca (Elliott, 1992). Desta forma, a crença dos entrevistados de que os pelicanos-pardos se alimentam de restos de peixes jogados na água por pescadores alinha-se com o descrito na literatura científica.

No Brasil, as aves são utilizadas para diferentes propósitos, sendo a alimentação um dos principais usos por populações tradicionais, que utilizam a carne, os ovos e mesmo os ossos destes animais (Alves et al., 2013; Soares et al., 2018). Tal como em outras áreas isoladas do país, na Amazônia brasileira a grande riqueza biológica e as condições socioeconômicas levaram as populações locais a desenvolver uma forte relação com os recursos faunísticos, o que resultou no desenvolvimento de um complexo sistema de informação sobre as espécies, traduzindo-se em saberes, crenças e práticas culturais (Santos-Fita et al., 2007). Nesta região, as populações humanas que habitam as áreas alagáveis têm desenvolvido há milênios uma expressiva relação de exploração dos recursos naturais (Vidal, 2008), onde a caça de animais silvestres é uma importante fonte de proteína, especialmente na época da cheia, quando a disponibilidade de peixes diminui (Calouro 1995; Pezzuti, 2009; Felix-Silva et al., 2018). A "caça de subsistência" de algumas espécies de aves associadas a ambientes aquáticos e também de migratórias na Amazônia é realizada por moradores das comunidades no entorno do Parque Nacional de Anavilhanas e do município de Novo Airão (Cintra et al., 2011). Este panorama amazônico pode explicar o abate de um pelicano-pardo, ainda que este seja uma ave exótica, rara e "diferente" para um morador local. No México, um dos mais frequentes usos culturais das aves é também a alimentação, tendo esses animais elevado valor na dieta de comunidades rurais, onde as grandes aves são as mais procuradas e frequentemente consumidas (Ávira-Nájera et al., 2020; Anderson et al., 2005).

Mostra-se bastante preocupante a oferta alimentar aos pelicanos-pardos devido ao potencial condicionamento das aves à aproximação humana. Quando não devidamente planejada e monitorada a oferta de alimentos a animais em vida livre pode trazer diversas consequências negativas, como mudança no estado comportamental, agressões por disputa de alimento com os outros animais e dependência (Orams, 2002). No Parque Nacional de Anavilhanas, assim como em outras áreas nos estados do Amazonas e Pará, já ocorre o turismo entre pessoas e botos (Inia geoffrensis) baseado na oferta alimentar aos cetáceos (Vidal et al., 2021). Durante muito tempo as interações com os botos foram realizadas sem quaisquer normas de monitoramento visando o bem-estar dos animas e a segurança dos turistas (Romagnoli, 2009; Vidal et al., 2013). Dessa forma, houve várias consequências negativas tanto para os botos quanto para os turistas, como número elevado de turistas em interação com poucos animais, turistas nadando com os botos e tentando segurá-los à força, oferta de alimentos que não faziam parte da dieta natural dos animais, tais como peixes congelados, cerveja, salsichas, pães, e nenhum controle da quantidade de peixes dada diariamente para cada boto (Romagnoli, 2009; Alves et al., 2011; Vidal et al., 2013). Segundo os pescadores de Novo Airão, o condicionamento dos botos à oferta alimentar vem causando uma maior aproximação dos cetáceos às redes de pesca, o que potencializa as capturas não intencionais dos animais e aumenta as chances de conflitos com os pescadores (Vidal et al., 2017); algo que também pode acontecer com os pelicanos-pardos que vem sendo condicionados. 


\section{Considerações Finais}

O registro de pelicanos-pardos no Parque Nacional de Anavilhanas e entorno nos meses de novembro de 2019 e fevereiro e março de 2020 apontam uma sazonalidade marcada, que coincide com o final da seca e início da enchente, respectivamente, na região do rio Negro. É possível que fatores climáticos de larga escala possam estar atuando sazonalmente e influenciando alguns indivíduos a se deslocarem muito de suas colônias e rotas migratórias usuais e demonstram que pouco sabemos sobre os fatores que influenciam em suas atividades de migração e dispersão. O monitoramento desses indivíduos por meio de anilhas ou de telemetria é importante para compreender melhor essas visitas inesperadas e identificar ainda se são os mesmos indivíduos que se fazem presentes.

Apesar de P. occidentalis ser classificada no status de "menos preocupante" na lista vermelha da IUCN, acredita-se que a sobreposição das irregulares atividades humanas que possam acontecer na região de Novo Airão, como a oferta alimentar desregrada e o abate intencional dos animais, seja por retaliação, seja para consumo, ameacem significativamente os indivíduos que possam se apresentar sazonalmente na região.

Entender as atitudes e os conhecimentos de populações tradicionais em relação aos recursos naturais representa fator chave para o sucesso de iniciativas conservacionistas. Assim, sugerimos aos gestores do Parque Nacional de Anavilhanas desenvolverem um adequado monitoramento das interações entre os pelicanos-pardos e os pescadores, complementado por eficientes estratégias que minimizem os impactos negativos nas aves, como ações de educação ambiental com o objetivo de inserir as comunidades em práticas que visem à conservação dos pelicanos-pardos, mas que também garantam o modo de vida tradicional dos moradores locais.

As ocasionais presenças destas exóticas aves podem ainda ser utilizadas como atrativo turístico por meio da observação à distância, sem oferta alimentar, e de modo a possibilitar aos visitantes compará-las com a rica avifauna nativa presente no Parque Nacional de Anavilhanas.

\section{Referências}

Almeida-Santos, D. A., Ferreira, G. S. \& Lopes, E. V. (2015). New record of the brown pelican Pelecanus occidentalis in continental waters of the Brazilian Eastern Amazon. Revista Brasileira de Ornitologia, 23, 351-353. https://doi.org/10.1007/bf03544304

Alves, L. C. P. S., Andriolo, A., Orams, M. B. \& Azevedo, A. F. (2011). The growth of "botos feeding tourism", a new tourism industry based on the boto (Amazon River dolphin) Inia geoffrensis in the Amazonas State, Brazil. Sitientibus Série Ciências Biológicas, 11(1), 8-15. http://dx.doi.org/10.13102/scb140

Alves, R. R. N., Silva, J. S., Silva Chaves, L. \& Albuquerque, U. P. (2018). Introduction - Ethnozoology Animals in Our Lives. In: Alves, R. R. N. \& Albuquerque, U. P. (Eds). Ethnozoology and Animal Conservation. Academic Press, p. 1-7.

Alves, R. R. N. \& Souto, W. M. S. (2015). Ethnozoology: A Brief Introduction. Ethnobiology and Conservation, 4, 1-13. https://doi.org/10.15451/ec2015-14.1-1-13

Alves, R. R. N., Leite, R. C. L., Souto, W. M. S., Bezerra, D. M. M. \& Loures-Ribeiro, A. (2013). Ethno-ornithology and conservation of wild birds in the semi-arid Caatinga of northeastern Brazil. Journal of Ethnobiology and Ethnomedicine, 9(14), 1-12. https://doi.org/10.1186/1746-4269-9-14

Anderson, E. N. \& Medina-Tzuc, F. (2005). Animals and the Maya in Southeast Mexico. University of Arizona Press.

Ávila-Nájera, D. M, Tigar, B. J., Zavala-Sánchez, Z., Zetina-Cordoba, P. \& Serna-Lagunes, R. (2020). A quantitative method for evaluating contemporary cultural uses of birds: a case study from Mexico. Ethnobiology Letters, 11(2), 5-19. https://doi.org/10.14237/ebl.11.2.2020.1644

Birdlife International. (2014). Medidas Mitigadoras da Captura Incidental - Informações práticas. ACAP Secretariat.

BirdLife International. (2018). Pelecanus occidentalis. The IUCN Red List of Threatened Species 2018: e.T22733989A132663224. https://dx.doi.org/10.2305/IUCN.UK.2018-2.RLTS.T22733989A132663224.en.

Blus, L. J., Lamont, T. G. \& Neely, B. S. (1979). Effects of organochlorine residues on eggshell thickness, reproduction, and population status of Brown Pelicans (Pelecanus occidentalis) in South Carolina and Florida, 1969-76. Pesticides Monitoring Journal, 12, $172-184$.

Brasil. (2011). Cadeia produtiva do turismo em Parques Nacionais no Brasil e entorno - Parque Nacional de Anavilhanas. SEBRAE/ICMBio.

Bugoni, L., Neves, T. S., Leite Jr., N. O., Carvalho, D., Sales, G., Furness, R. W., Stein, C. E., Peppes, F. V., Giffoni, B. B. \& Monteiro, D. S. (2008). Potential bycatch of seabirds and turtles in hook-and-line fisheries of the Itaipava Fleet, Brazil. Fisheries Research, 90(1-3), 217-224. https://doi.org/10.1016/j.fishres.2007.10.013 
Calouro, A. M. (1995). Caça de subsistência, sustentabilidade e padrões de uso entre seringueiros ribeirinhos e não ribeirinhos do Estado do Acre. 82p. Dissertação (Mestrado em Ecologia) - Universidade de Brasília, Brasília.

Cintra, R. \& Rosas, M. (2011). Parque Nacional de Anavilhanas. In: Valente, R. M., Silva, J. M. C., Straube, F. C. \& Nascimento, J. L. X. (Org.). Conservação de aves migratórias neárticas no Brasil. Conservação Internacional, p. 55-58.

Collazo, J. A. (1985). Status and ecology of the Brown Pelican in the Greater Puerto Rican Bank region. 201p. Thesis (Doctorate in Animal Ecology) - Iowa State University, Ames. https://doi.org/10.31274/rtd-180813-8681

Costa-Neto, E. M., Vargas Clavijo, M. \& Santos Fita, D. (2009). Manual de Etnozoología. Una Guía Teórico Práctica Para Investigar la Interconexión del Ser Humano con los Animales. Tundra Ediciones.

Crouch, M. \& Mckenzie, H. (2006). The logic of small samples in interview-based qualitative research. Social Science Information, 45, 483-499. https://doi.org/10.1177/0539018406069584

Del Hoyo, J., Elliot, A. \& Sargatal, J. (1992). Handbook of the Birds of the World, Vol. 1: Ostrich to Ducks. Lynx Edicions.

Elliott, A. (1992). Family Pelecanidae. In: Del Hoyo, J., Elliott, A. \& Sargatal, J. (Eds.). Handbook of the Birds of the World, Vol. 1: Ostrich to Ducks. Lynx Edicions, p. 290-311.

Elliott, A., Christie, D. A., Jutglar, F., Juana, E. \& Kirwan, G. M. (2018). Brown Pelican (Pelecanus occidentalis). In: Del Hoyo, J., Elliott, A., Sargatal, J., Christie, D. A. \& De Juana, E. (Eds.). Handbook of the Birds of the World. Lynx Edicions.

Enticott, J. \& Tipling, D. (1997). Seabirds of the world: the complete reference. Stackpole Books.

Franson, J. C, Hansen, C. P, Creekmore, T. E, Brand, C. J, Evers, D. C, Duerr, A. E. \& Destefano, S. (2003). Lead Fishing Weights and Other Fishing Tackle in Selected Waterbirds. Waterbirds, 26(3), 345-352. https://doi.org/10.1675/1524-4695(2003)026[0345:1fwaof]2.0.co;2

Felix-Silva, D., Vidal, M. D., Alvarez Junior, J. B. \& Pezzuti, J. C. B. (2018). Caracterização das atividades de caça e pesca na Floresta Nacional de Caxiuanã, Pará, Brasil, com ênfase no uso de quelônios. Biodiversidade Brasileira, 8, 232-250.

Ferreira, E. C., Muelbert, M. M. C. \& Secchi, E. R. (2010). Distribuição espaço-temporal das capturas acidentais de toninhas (Pontoporia blainvillei) em redes de emalhe e dos encalhes ao longo da costa sul do Rio Grande do Sul, Brasil. Atlântica, 32(2), 183-197. https://doi.org/10.5088/atl.2010.32.2.183

Filgueira, C. H. M. S., Zappes, C. A., Vidal, M. D. \& Nunes, J. L. S. (2021). Traditional knowledge of artisanal Fishers and Sotalia guianensis (Van Bénéden, 1864) (Cetacea, Delphinidae) in the Extractive Reserve Baía do Tubarão (Brazilian Amazon coast). Ocean \& Coastal Management, 210: 105700. https://doi.org/10.1016/j.ocecoaman.2021.105700

Fogarty, M. J., Nesbitt, S. A. \& Gilbert. C. R. (1981). Diet of nestling Brown Pelicans in Florida. Florida Field Naturalist, 9, 38-40.

Gil, A. C. (2008). Métodos e técnicas de pesquisa social. (6a ed.), Atlas.

Goldenberg, M. (1999). A arte de pesquisar: como fazer pesquisa qualitativa em Ciências Sociais. Record.

Gomes, F. B. R., Bernardes, V. C. D., Lacava, R., Lopes Baptista, L. A. M. \& Oliveira e Silva, M. B. (2016). Records of brown pelican Pelecanus occidentalis inland in Northern Brazil: distributional expansion or juvenile dispersal? Marine Ornithology, 44, $247-248$.

Goulding, M., Carvalho, M. L. \& Ferreira. E. G. (1988). Rio Negro, rich life in poor water. SPB Academic Publishing.

Grzimek, B. (2002). Animal life encyclopedia. Thomson-Gale.

Illenseer, R. \& Pereira, H. S. (2011). Mosaicos fluídos: estudo de caso da relação da pesca e áreas protegidas no Mosaico do Baixo Rio Negro (AM). In: Anais do V Seminário Brasileiro sobre Áreas Protegidas e Inclusão Social. Editora da UFAM.

Mason, M. (2010). Sample Size and Saturation in PhD Studies Using Qualitative Interviews. Forum: Qualitative Social Research, 11(3), Art. 8. https://doi.org/10.17169/fqs-11.3.1428

Mazzochi, M. S. \& Carlos, C. J. (2020). Pescadores e aves marinhas: etnobiologia de uma comunidade pesqueira no Sul do Brasil. Biotemas, 33(2), 1-16. https://doi.org/10.5007/2175-7925.2020.e71987

Mitchell, M. H. (1957). Observations on Birds of Southeastern Brazil. University of Toronto Press.

Neves, T. S., Olmos, F., Peppes, F. V. \& Mohr, L. V. (2006). Plano de ação para a conservação de albatrozes e petréis. https://www.icmbio.gov.br/portal/images/stories/\%20docs-plano-de-acao/pan-albatrozes/albatrozes-parte1.pdf.

Opdenakker, R. (2006). Advantages and disadvantages of four interview techniques in qualitative research. Forum: Qualitative Social Research, 7(4), Art. 11. http://nbn-resolving.de/urn:nbn:de:0114-fqs0604118

Orams, M. B. (2002). Feeding wildlife as a tourism attraction: issues and impacts. Tourism Management, 23(3), 281-293. https://doi.org/10.1016/S02615177(01)00080-2

Palmer, R. S. (1962). Handbook of North American Birds. Yale University Press.

Patrial, L. W., Pessoa, A. S. P. \& Pereira, G. A. (2011). Primeiro registro do pelicano-peruano Pelecanus thagus no Brasil e registro documentado do pelicanopardo P. occidentalis na costa leste brasileira. Revista Brasileira de Ornitologia, 19, 539-540. 
Pereira, A. S., Shitsuka, D. M., Parreira, F. J. \& Shitsuka, R. (2018). Metodologia da pesquisa científica. UFSM.

Peres, C. A. (2005). Por que precisamos de megareservas na Amazônia. Megadiversidade, 1(1), 174-180.

Peterson, R. T. (2008). Peterson field guide to birds of North America. Houghton Mifflin Co.

Pezzuti, J. C. B. (2009). Manejo de caça e a conservação da fauna silvestre com participação comunitária. Paper do NAEA, 235,1 -13.

Pinto, O. M. O. (1978). Novo Catálogo das aves do Brasil, la parte. Empresa Gráfica da Revista dos Tribunais.

Prince, P. A. \& Morgan, R. A. (1987). Diet and feeding ecology of Procellariiformes. In: Croxall, J. P. (Ed.). Seabirds: feeding biology and role in marine ecosystems. Cambridge University Press, p. 135-173.

Rodrigues, F. M. G. (2014). Unidades de conservação, pesca e modo de vida: Contradições. 123p. Dissertação (Mestrado em Geografia) - Universidade Federal do Amazonas, Manaus.

Romagnoli, F. C. (2009). Interpretação ambiental e envolvimento comunitário: ecoturismo como ferramenta para a conservação do boto-vermelho, Inia geoffrensis. 133p. Dissertação (Mestrado em Biologia de Água Doce e Pesca Interior) - Universidade Federal do Amazonas/Instituto Nacional de Pesquisas da Amazônia, Manaus.

Santos, G. M., Ferreira, E. J. G. \& Zuanon, J. A. S. (2006). Peixes comerciais de Manaus. Ibama/ProVárzea.

Santos-Fita, D. \& Costa-Neto, H. M. (2007). As interações entre os seres humanos e os animais: a contribuição da etnozoologia. Biotemas, 20(4), 99-110.

Schensul, S. L., Schensul, J. J. \& Lecompte, M. D. (1999). Essential Ethnographic Methods: Observations, Interviews and Questionnaires. Altamira Press.

Shields, M. (2002). Brown Pelican (Pelecanus occidentalis): The Birds of North America Online. https://www.allaboutbirds.org/guide/brown_pelican/lifehistory.

Sick, H. (1997). Ornitologia Brasileira. Editora Nova Fronteira.

Silva, A. H. \& Fossá, M. I. T. (2015). Análise de conteúdo: exemplo de aplicação da técnica para a análise de dados qualitativos. Revista Eletrônica Qualitas, 16(1), 1-14.

Silva, M. A. \& Simonetti, S. R. (2020). Avaliação dos atrativos turísticos do Parque Nacional de Anavilhanas (AM). Revista Brasileira de Ecoturismo, 13(1), 69-87. https://doi.org/10.34024/rbecotur.2020.v13.6791

Snethlage, E. (1914). Catálogo das aves amazônicas. Boletim do Museu Paraense Emílio Goeldi, 8, 1-465.

Soares, V. M. S., Soares, H. K. L., Santos, S. S. \& Lucena, R. F. P. (2018). Local knowledge, use, and conservation of wild birds in the semi-arid region of Paraíba state, northeastern Brazil. Journal of Ethnobiology and Ethnomedicine, 14, 1-13. https://doi.org/10.1186/s13002-018-0276-x

Tangley, L. (2009). Oil Spill Hammers Brown Pelicans. National Wildlife, 48(6), 12-14.

Teixeira, D. M., Otoch, R., Luigi, G., Raposo, M. A. \& Almeida, C. C. (1993). Notes on some birds of northeastern Brazil (5). Bulletin of the British Ornithologists' Club, 113, 48-52.

Vargas-Masís, R. \& Arguedas-Rodrigues, P. (2014). Firts record of leucism in brown pelicans (Pelecanus occidentalis) in Costa Rica. Revista Ciencias Marinas Y Costeras. 6, 149-154. https://doi.org/10.15359/revmar.6.10

Vidal, M. D. (2008). Experiências de manejo dos recursos naturais na várzea amazônica. Ibama/ProVárzea.

Vidal, M. D., Santos, P. M. C., Oliveira, C. V. \& Melo, L. C. (2013). Perfil e percepção ambiental dos visitantes do flutuante dos botos, Parque Nacional de Anavilhanas, Novo Airão - AM. Revista Brasileira de Pesquisa em Turismo, 7(3), 419-435. https://doi.org/10.7784/rbtur.v7i3.583

Vidal, M. D., Alves, L. C. P. S., Zappes, C. A., Andriolo, A. \& Azevedo, A. F. (2017). Percepção de pescadores sobre as interações de botos com a pesca e sua relação com o turismo de alimentação artificial em Novo Airão, Amazonas, Brasil. In: Marchand. G. \& Vander Velden, F. (Orgs.). Olhares cruzados sobre as relações entre seres humanos e animais silvestres na Amazônia (Brasil, Guiana Francesa). EDUA, p. 103-120.

Vidal, M. D., Chaves, E. P. \& Silva, V. A. R. (2019). Biotecnologia como ferramenta para o conhecimento e conservação da fauna e flora amazônica. In: Campos, M. A. \& Maia, R. T. (Orgs.). Genética e melhoramento de plantas e animais. Atena Editora, p. 78-87. https://doi.org/10.22533/at.ed.1921917109

Vidal, M. D., Santos, P. M. C., Chaves, M. P. S. R. \& Swett, R. (2021). Challenges and advances in the planning of tourism with Amazon River dolphins in the Brazilian Amazon. In: Khan, S.A.R. (Org.). Tourism. 1 ed., IntechOpen, p. 1-16. https://doi.org/10.5772/intechopen.93894

Vooren, C. M. \& Fernandes, A. C. (1989). Guia de albatrozes e petréis do sul do Brasil. Sagra.

Voous, K. H. (1983). Birds of the Netherlands Antilles. De Walburg Press. 